Conclusion Health impact assessment is a new science, and complexities involved in assessing new drugs in the global context are formidable. However, initial models suggest that the HIF could significantly change the focus of drug innovation. Only pilot studies will properly test the HIF's underlying principles and uncover the practical challenges which will determine its implementation and effectiveness. The creation of the HIF could bring great advances in epidemiological data collection and its application.

\section{P1-10 DEVELOPMENT AND PSYCHOMETRIC PROPERTIES OF A SCALE TO MEASURE HEALTH EFFECTS OF DISCRIMINATION}

doi:10.1136/jech.2011.142976c.5

\begin{abstract}
1J Bastos, ${ }^{*}{ }^{2} E$ Faerstein, ${ }^{3} \mathrm{R}$ Celeste, ${ }^{1} \mathrm{~A}$ Barros. ${ }^{1}$ Federal University of Santa Catarina, Florianopolis, Santa Catarina, Southern Brazil, Brazil; ${ }^{2}$ State University of Rio de Janeiro, Rio de Janeiro, Southeastern Brasil, Brazil; ${ }^{3}$ Federal University of Rio Grande do Sul, Porto Alegre, Rio Grande do Sul, Southern Brazil, Brazil;, ${ }^{4}$ Federal University of Pelotas, Pelotas, Rio Grande do Sul, Southern Brazil, Brazil
\end{abstract}

Introduction The development of discrimination scales is an emerging field of enquiry in the area of social determinants of health. However, published scales cannot distinguish health consequences of discrimination as a result of the exposure to differential treatments of any kind from the strict attribution of these events to discrimination. We report the development of a scale that may clarify the relative importance of the effects of these cognitive mechanisms for health outcomes.

Methods Successive versions of the instrument were developed based on a systematic review of racial discrimination scales, focus groups and an evaluation by a panel of experts. Instrument refinement was achieved via cognitive interviews and pilot-testing, so that the final scale version was administered to 424 university students in Rio de Janeiro, Brazil. Structural dimensionality, two types of reliability and construct validity were assessed.

Results Exploratory factor analysis corroborated the hypothesis of unidimensionality, and the experts indicated that scale items were face and content valid. Internal consistency, estimated by Cronbach's $\alpha$, was 0.8 , and test-retest reliability was higher than 0.5 for 14 out of the 18 items, according to the weighted $\kappa$ statistics. The scale's score was statistically higher among socially disadvantaged individuals and correlated with adverse health behaviours and outcomes. Nevertheless, the low test-retest reliability and the observed invariance of specific items indicate that the scale should be assessed in other population domains.

Conclusion The proposed scale will enable the investigation of aspects of the relationship between discrimination and health not previously documented in the literature.

\section{P1-11 WITHDRAWN}

\section{P1-12 DETECTING DIFFERENCES BETWEEN TREATMENTS IN CRITICAL CARE TRIALS USING MIXTURES OF PARAMETRIC SURVIVAL DISTRIBUTIONS}

doi:10.1136/jech.2011.142976c.6

${ }^{1}$ W Checkley, ${ }^{* 1} \mathrm{R}$ Brower, ${ }^{2} \mathrm{~A}$ Muñoz. ${ }^{1}$ Division of Pulmonary and Critical Care, Johns Hopkins University, Baltimore, Maryland, United States Minor Outlying Islands; ${ }^{2}$ Department of Epidemiology, Johns Hopkins University, Baltimore, Maryland, USA

Background Traditional methods in survival analysis inadequately summarise the timing of outcomes in critical care trials because they accommodate only one clinical endpoint. We sought to develop an analytical approach to detect differences when there are two clinical endpoints where one may preclude the other.

Methods We used a mixture of parametric survival distributions that belong to the three-parameter generalised $\gamma$ family (generalised $\gamma, \gamma$, Weibull, log-normal, and exponential) to model the timing and frequency of two clinical endpoints jointly. Study outcomes were hospital mortality at 60 days and time to unassisted breathing (UAB) at 28 days. We used data from a trial of methylprednisolone vs placebo in 180 critically ill patients with persistent ARDS to show our approach.

Results The best model to fit these data was a mixture of lognormal distributions. Patients who received methylprednisolone achieved UAB earlier than did those who received placebo $(p=0.05)$; however, this effect decreased over time: by day $5,55 \%$ more patients (95\% CI $16 \%$ to $79 \%$ ) achieved UAB in the methylprednisone group while by day $20,25 \%$ more patients $(95 \%$ CI $6 \%$ to $42 \%$ ) achieved UAB. The overall probability of achieving UAB was similar between both study groups $(p=0.82)$, as were the times to death $(\mathrm{p}=0.15)$.

Conclusions Times of $\mathrm{UAB}$ between the study groups were not proportional over time and are unlikely to be proportional in any trial where duration of mechanical ventilation is affected. Furthermore, our approach can easily accommodate mixtures of several well-known parametric distributions under a single comprehensive family, which simplifies hypothesis testing.

\section{P1-13 MEAT INTAKE AND URINARY TRACT TUMOURS RISK ASSESSMENT THROUGH PROMOTING LATENT VARIABLES MODELS}

doi:10.1136/jech.2011.142976c.7

${ }^{1} \mathrm{~S}$ E Muñoz, ${ }^{*} \mathrm{M} \mathrm{D}$ Román, ${ }^{2} \mathrm{~A}$ Navarro, ${ }^{3} \mathrm{~L} \mathrm{R}$ Aballay, ${ }^{3} \mathrm{M}$ del P Díaz. ${ }^{1}$ CONICET, Cellular Biology Institute, Faculty of Medical Sciences, University of Córdoba, Córdoba, Argentina; ${ }^{2}$ School of Nutrition, Faculty of Medical Sciences, University of Córdoba, Córdoba, Argentina; ${ }^{3}$ Biostatistics Unit, School of Nutrition, Faculty of Medical Sciences, University of Córdoba, Córdoba, Argentina

Introduction Generalize Linear Latent and Mixed Models are scarcely used in cancer epidemiology, having been basically used multilevel and generalised linear and mixed models. Using flexible models allow including random effects, common factors coupled to a multilevel structure for unobserved heterogeneity. Cancer is the main cause of death worldwide. In Córdoba, (Argentina) bladder cancer is the fourth most incident cancer among men and ninth in the overall population. Previous studies have suggested that fruits, lean meats, some cereals and cereal products, and vegetable oils would prevent against these tumours, while some fatty meats and use of sweeteners, may increase the risk. In the present work new methodological strategies are used in order to explore the dietary influence on the disease outcome.

Objective To define some possible promoting dimensions related to meat intake and combine with a disease model including some biosocio-cultural characteristics.

Methods A case-control study, conducted in Córdoba, including 221/ 472 cases/controls is used. Subjects were interviewed using a validated FFO containing biological, educational and lifestyle characteristics, and 127 food items. A two steps model was proposed: common factor modelling as confirmatory factor analysis to explore the dimensionality of constructs from the diet information; and a disease model, which arises from the composition of exposure and measurement models

Results and Conclusion Two constructs were identified, a promoting and a protective one. The direct and indirect covariates effects were also estimated as risk. This work improves the understanding about the diet-cancer relationship. 